\title{
Texture analysis using complex system models: fractal dimension, swarm systems and non-linear diffusion
}

\author{
Bruno Brandoli Machado ${ }^{1}$, Jose Fernando Rodrigues Junior ${ }^{1}$ \\ ${ }^{1}$ University of São Paulo (ICMC/USP) \\ 13566-590, São Carlos, SP, Brasil
}

\begin{abstract}
brunobrandoli@gmail.com, juniodicmc.usp.br
Abstract. Texture is one of the primary visual features used to computationally describe the patterns found in nature. Existing computational methods, however, do not successfully discriminate the complexity of texture patterns. Such methods disregard the possibility of describing images by benefiting from the complex systems properties that are characteristic to textures. To do so, we created approaches based on the Bouligand-Minkowski fractal dimension, swarm-system Artificial Crawlers, and non-linear diffusion of Perona-Malik, techniques that led to methodologies with efficacy and efficiency comparable to the state-ofthe-art. The results achieved in the four methodologies described in this work demonstrated the validity and the potential of our hypothesis in tasks of pattern recognition. The contributions of our methodologies shall support advances in materials engineering, computer vision, and agriculture.
\end{abstract}

\section{Introduction}

Texture is an important visual attribute in computer vision with many areas of applications. Recently, texture analysis has been widely applied to remote sensing [1], industrial inspection [2], medical image analysis [3], face recognition [4], among many others. Although the human visual system can easily discriminate textural patterns, the description by automatic methods has been a great challenge. Indeed, there is no universally accepted definition of texture. It is usually referred to as a repetitive pattern that can vary according to the size, which produces different tactile sensations associated with roughness, coarseness, and regularity. Furthermore, texture patterns are related to the physical properties of surfaces present in images, making them a powerful tool for image analysis.

The proposed methods have been grouped according to the mathematical aspects used to handle the patterns present in the images. There are five major categories: structural, statistical, spectral, model-based, and agent-based. The structural methods rely on primitives that provide a symbolic description of the images [5]. The idea comes from concepts on mathematical morphology, which describes an image by evolving morphological operations with different sizes of structuring elements, a useful technique to handle shapes in textures. Statistical methods represent textures by the spatial distribution of the gray-level pixels in the image. One of the best methods of this category, and still very popular, is co-occurrence matrices [6]. In the same line, Dmitry Chetverikov [7] introduced the technique named interaction map. Similarly, Ojala et. al. [8] proposed a method that describes images based on the occurrence of gray values on circular local neighborhoods; it is named local binary patterns (LBP). Xiaoyang et al. [9] extended the idea of LBP to local ternary pattern (LTP), which considers the magnitude of pixel derivatives along with its sign to generate the ternary code.

Model-based methods were proposed with the assumption that textures can be represented by mathematical models, including stochastic models of Markov random fields 
[10] and fractality [11]. Particularly, fractal geometry has drawn great attention on the task of describing textures. The main reason is because fractal geometry is able to describe irregular or fragmented shapes of natural phenomena, as well as other complex objects that traditional Euclidean geometry is not able to describe. The fractal concept is expressed by the time-domain statistical scaling laws and is characterized by the powerlaw behavior of physical systems. This idea makes use of the geometrical interpretation of objects and takes into account the degree of regularity of the structure related to its physical behavior [11].

Most of the methods used in texture analysis are restricted to the analysis of the spatial relations over small neighborhoods, or they are based on the extraction of global features of the whole image on a single scale. As a consequence, they do not perform well in high-complex geometry textures. In contrast, spectral methods or signal processing methods, including Fourier analysis [12], Gabor filters [13], wavelet transform methods [14], were developed inspired by evidence that the human visual system describes images by the frequency domain. However, the Fourier transform lacks spatial information, what impairs its potential for image description. Although Gabor filters present joint image resolution in both the spatial and frequency domains, they do not describe well coarse textures; this is because the energy of such textures is concentrated in subimages of lowest frequencies. In contrast with Gabor filters, wavelets decompose a texture image into a set of frequency channels. However, wavelet analysis is basically a linear analysis and suffers from uniformly-poor resolutions over different scales and from its non-data adaptive nature, since the same wavelet basis is used to analyze all the data.

\section{Goals and Contributions}

The goal of this thesis is to propose solutions to overcome the drawbacks of current texture analysis methods. To reach this goal, we have proposed new methods for texture description based on complex systems, including fractal dimension, swarm systems, non-linear diffusion models, and complex networks. We have addressed two main issues found in typical statistical and model-based texture descriptors: (1) the lack of a multiscale representation to capture the richness of local features in different levels of observation; and, (2) although fractal descriptors proved to be a promising texture descriptor, current methods do not explicitly consider the neighborhood relation in terms of the gray levels of the texture. In this work, we evaluated our methods over four well-known texture benchmarks: Brodatz, Vistex, Outex and Usptex. In addition, our methods were compared with state-of-the-art texture methods. The motivation to develop our methods is that they are suitable for real-world applications, as in material engineering and agriculture, leading to better or automatic decisions. As we show along the text, we focus on nanomaterial quality assessment and on plant disease identification in soybean leaves.

\subsection{Theoretical Contributions}

The first contribution of this work is a methodology for texture description. We surpass the state-of-the-art, as proposed by Zhang and Chen [15], by introducing a new rule of movement that considers both high and low-level intensities on images using the swarm system named artificial crawler. We developed an improved method for assessing the quality of the silk fibroin scaffolds with two rules of movement: maxima and minima. Our goal is to provide an effective method to support visual analysis, thus reducing the subjectivity of the human analysis. We evaluated the potential of the silk fibroin by including glycerol in the solution during scaffold formation. This work was published in the Computational Science and Discovery Journal [16]. 
The second contribution of this thesis is an extended with the BouligandMinkowski fractal dimension method. The methodology is based on the energy information of the artificial crawler swarm system extracted from two rules of movement. Similar to the method proposed in the first contribution, each agent is able to move to the higher intensities, as well as to lower ones. Although we can find the minima and maxima of images directly, the underlying idea is to characterize the path of movement during the evolution process. Our method differs from our first contibution since we quantify the state of the swarm system after stabilization by employing the Bouligand-Minkowski fractal dimension method. In the method, the energy information was considered the most important attribute due to its capacity of representing the interaction between the movement of agents and the environment. This work was published in the journal Physica A: Statistical Mechanics and its Applications [17] and, an example of the path configuration for 1,000 artificial crawlers is presented in Figure 1 .

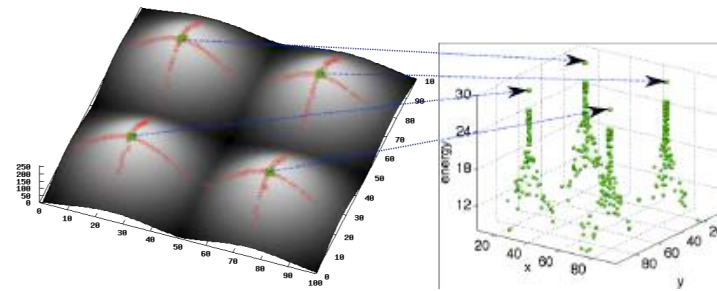

(a) $r=1$

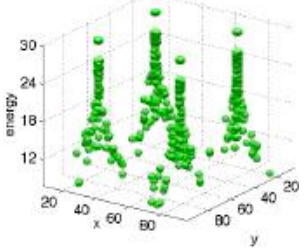

(b) $r=2$

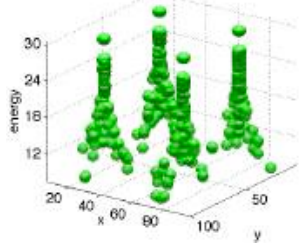

(c) $r=3$

Figure 1. (a) An image example and the mapping of artificial crawler agents, after convergence, onto a 3D space, (b) and (c) show an illustration of the dilation process for the fractal dimension of the swarm system artificial crawlers.

The third contribution of this thesis is a multiscale texture descriptor based on non-linear diffusion designed to better represent textures in the task of image classification. Many recent texture-analysis methods are developed to extract measures on a single scale. In contrast, we assume that an image texture reveals different structures according to the scale of observation, so that the scale concept of multiscale representation is of crucial importance [18]. Thus, we iteratively represent the original image in a set of new images, where images are combinations of both oscillatory (texture) and geometrical (cartoon) patterns, we get two components for the derivative images. At each iteration step, we estimate the average and deviation of the descriptors computed over the two components. We then combine the measures from both components to compose our feature vector. The fractal dimension is adopted here due to its precision in quantifying structural properties. Experimental results over four well-known texture datasets reveal a superior performance of our method. This work was first published in the Proceedings of the 13th International Conference on Advanced Concepts for Intelligent Vision Systems with Gabor filters [19]. Later, a new research involving fractal descriptors was submitted to the Pattern Recognition Letters Journal [18] and the main ideia of the proposal is presented in Figure 2 .

\subsection{Applied Contributions}

The fourth contribution of this work is the application of image analysis methods in an innovation project that resulted in a software registration deposit, in a commercial application (available at the GooglePlay store), and on a publication in the Computer Electronics and Agriculture Journal [20]. In this case, we assume that a leaf image presents 


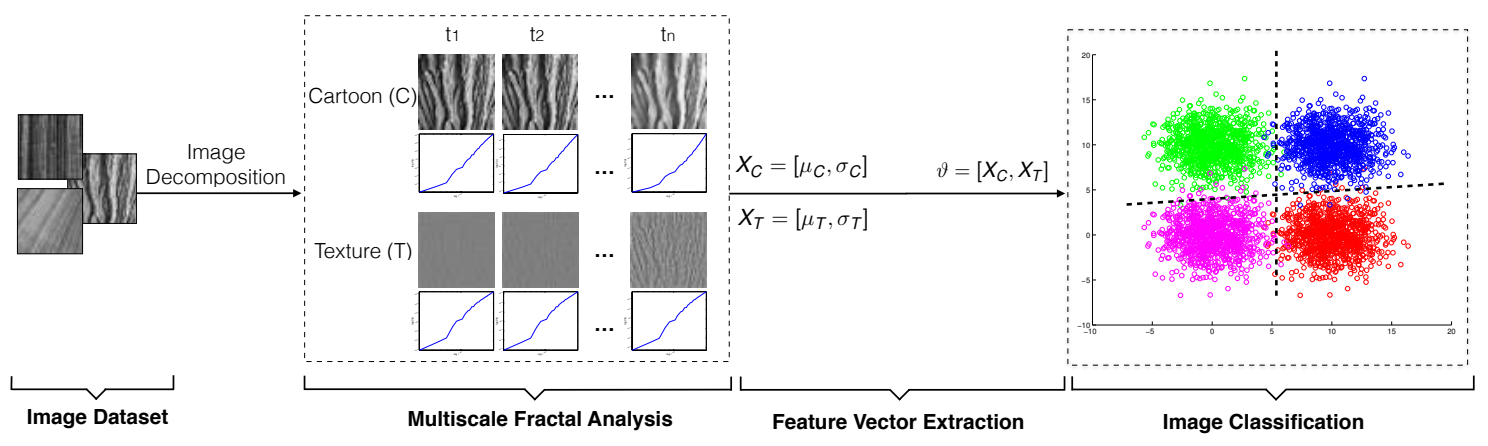

Figure 2. Schematic representation of our multiscale proposal.

different details according to the scale of analysis, which is suitable to describe fractallike structures as observed in leaves. The texture analysis has been integrated to the mobile application named BioLeaf - Foliar Analysis. In the same line and with fractal dimension, we have been used it to measure the spraying coverage to assess the quality of pesticide spraying machines, an application named DropLeaf - Deposition Analysis. This mobile application is in a commercial negotiation. We invite the reader to visit the BioLeaf and at DropLeaf website at http://bioleaf.icmc.usp.br and http://dropleaf.icmc.usp.br, which present all the details of the applications.
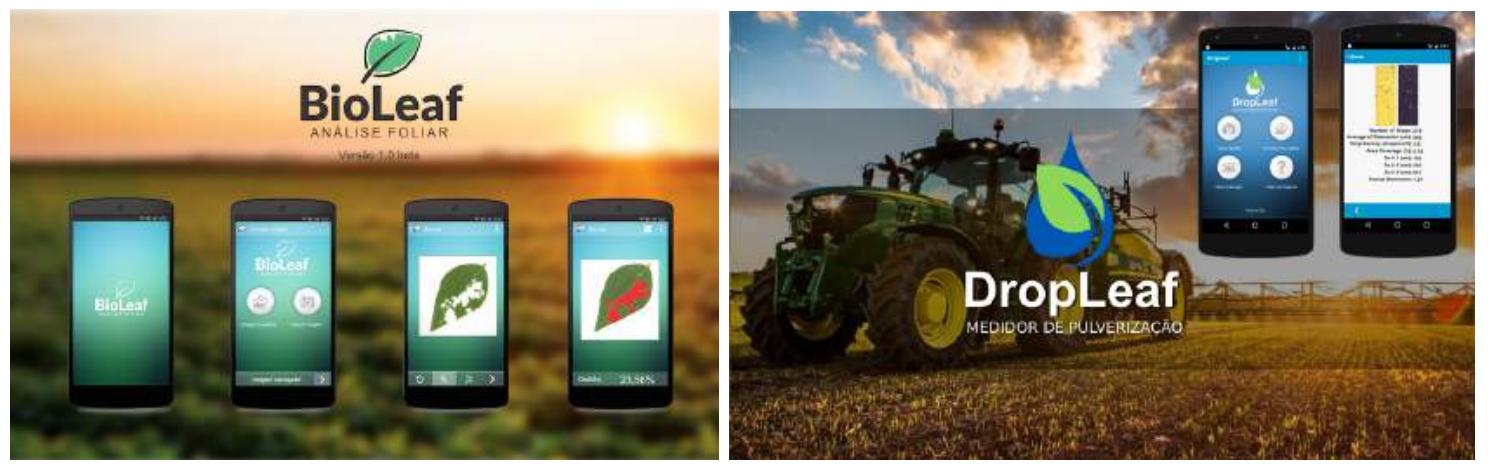

Figure 3. (a) Screenshots of BioLeaf - Foliar Analysis and, in (b) screenshots of DropLeaf - Deposition Analysis.

The fifth contribution of this thesis is a complex network approach for particle agglomeration analysis in nanoscale images. In this work, instead of assuming that a nanoscale image is a textured surface, we have modeled the nanoparticles like vertices of a graph, while connections are created according to a thresholding for density estimation over a certain radius. For each nanoparticle, we calculate its density. Two particles are linked, defining and edge, only if their distance is smaller than a given radius and its density is higher than a given threshold. This work was published in the Journal of Nanoparticle Research [21]. Furthermore, this work was integrated into an expert system (see Figure 4), named NanoImage Analyzer, and it was submitted for software registration also at INPI.

\section{Conclusion}

Texture description has always been a challenging task in image analysis and computer vision. Texture analysis methods have emerged as important tools for real-world appli- 


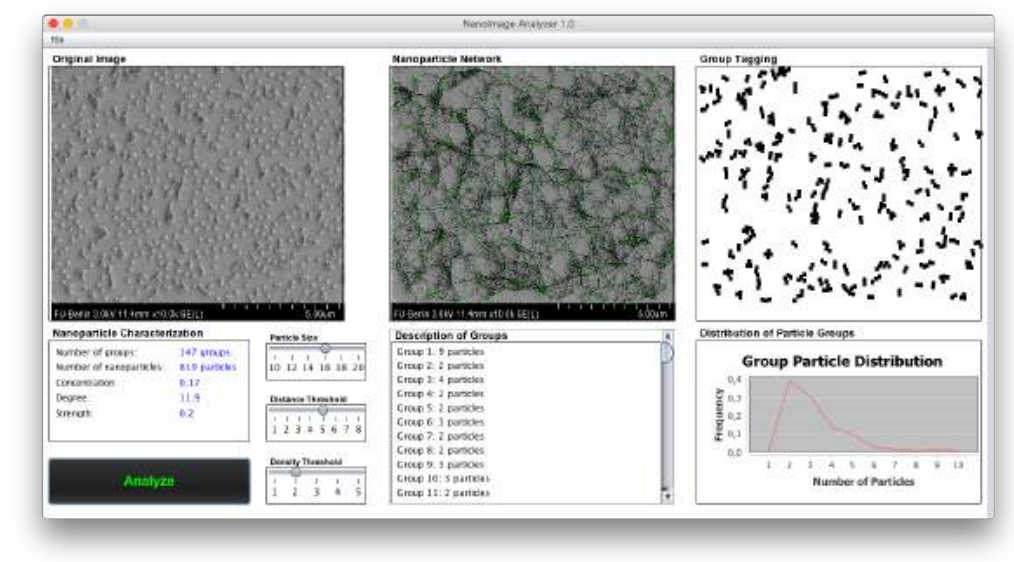

Figure 4. Tool NanolmageAnalyzer for analysis of nanoparticles agglomeration. It can be downloaded freely at http://nanoanalyzer.icmc.usp.br

cations, this is because they can learn patterns from objects or regions with annotated examples. Typically, such methods aim to measure raw image pixels into a discriminant data space. Despite the achievements, effective feature description is still a challenge. In this work, we introduced novel methods for texture description applied over domains ranging from agriculture to nanomaterials. We have achieved promising results, as previously discussed, with the aim of demonstrating that texture, as found in nature, is of great potential in image analysis and computer vision.

In conclusion, this work departed from the hypothesis that the use of texture information can improve systems that depend on image analysis. We tested this initial assumption over several domains using different proposed methods. Our results, as discussed in the previous section, demonstrated that, indeed, multiscale image representation has potential in enhancing the discriminatory power when the scale is taken into account. Future directions of the feature design are lead by advances in deep neural network construction, that has brought a significant breakthrough for feature description, however, our preliminary experiments showed that it is needed a large number of training samples for achieving good results.

\section{References}

[1] M. Gong, Y. Li, L. Jiao, M. Jia, and L. Su, "Sar change detection based on intensity and texture changes," Journal of Photogrammetry and Remote Sensing, vol. 93, no. 0, pp. 123-135, 2014.

[2] C. S. Tsang, H. Y. Ngan, and G. K. Pang, "Fabric inspection based on the elo rating method," Pattern Recognition, vol. 51, no. 3, pp. 378-394, 2016.

[3] N. Zaglam, P. Jouvet, O. Flechelles, G. Emeriaud, and F. Cheriet, "Computer-aided diagnosis system for the acute respiratory distress syndrome from chest radiographs," Computers in Biology and Medicine, vol. 52, no. 0, pp. 41-48, 2014.

[4] R. Mehta, J. Yuan, and K. Egiazarian, "Face recognition using scale-adaptive directional and textural features," Pattern Recognition, vol. 47, no. 5, pp. 1846-1858, 2014.

[5] Y. Chen and E. Dougherty, "Gray-scale morphological granulometric texture classification,” Optical Engineering, vol. 33, no. 8, pp. 2713-2722, 1994.

[6] R. M. Haralick, "Statistical and structural approaches to texture," Proceedings of the IEEE, vol. 67, no. 5, pp. 786-804, 1979. 
[7] D. Chetverikov, "Texture analysis using feature based pairwise interaction maps," Pattern Recognition, vol. 32, pp. 487-502, March 1999.

[8] T. Ojala, M. Pietikäinen, and T. Mäenpää, "Multiresolution gray-scale and rotation invariant texture classification with local binary patterns," IEEE Trans. Pattern Anal. Mach. Intell., vol. 24, pp. 971-987, July 2002.

[9] X. Tan and B. Triggs, "Enhanced local texture feature sets for face recognition under difficult lighting conditions," Image Processing, IEEE Transactions on, vol. 19, pp. 1635-1650, June 2010.

[10] G. R. Cross and A. K. Jain, "Markov random field texture models," IEEE Trans. Pattern Anal. Mach. Intell., vol. 5, pp. 25-39, 1983.

[11] C. Tricot, Curves and fractal dimension. Springer-Verlag, 1995.

[12] R. Azencott, J.-P. Wang, and L. Younes, "Texture classification using windowed fourier filters," IEEE Trans. Pattern Anal. Mach. Intell., vol. 19, pp. 148-153, February 1997.

[13] D. Gabor, "Theory of communication," Journal of Institute of Electronic Engineering, vol. 93, pp. 429-457, November 1946.

[14] S. Mallat and S. Zhong, "Characterization of signals from multiscale edges," IEEE Trans. Pattern Anal. Mach. Intell., vol. 14, no. 7, pp. 710-732, 1992.

[15] D. Zhang and Y. Q. Chen, "Artificial life: a new approach to texture classification," International Journal of Pattern Recognition and Artificial Intelligence, vol. 19, no. 3, pp. 355-374, 2005.

[16] B. B. Machado, W. N. Goncalves, and O. M. Bruno, "Artificial crawler model for texture analysis on silk fibroin scaffolds," Computational Science and Discovery, vol. 0, no. 7, p. 015004, 2014.

[17] W. N. Goncalves, B. B. Machado, and O. M. Bruno, "Texture descriptor combining fractal dimension and artificial crawlers," Physica A: Statistical Mechanics and its Applications, vol. 395, pp. 358-370, 2014.

[18] B. B. Machado, W. N. Goncalves, M. do Santos, and J. F. R. Jr., "Multiscale fractal description using non-liner diffusion of perona-malik for texture analysis," Pattern Recognition Letters, 2016.

[19] B. B. Machado, W. N. Goncalves, and O. M. Bruno, "Enhancing the texture attribute with partial differential equations: a case of study with gabor filters," in Proceedings of the 13th international conference on Advanced concepts for intelligent vision systems, ACIVS'11, pp. 337-348, Springer-Verlag, 2011.

[20] B. B. Machado, J. Orue, M. dos Santos, D. Sarath, G. Goncalves, W. N. Goncalves, H. Pistori, R. R. Mauro, and J. F. R. Jr., "Bioleaf: a professional mobile application to measure foliar damage caused by insect herbivory," Computer Electronics and Agriculture, vol. 129, no. 3, pp. 44-55, 2016.

[21] B. B. Machado, L. Scabini, M. do Santos, W. N. Goncalves, R. Moraes, and J. F. R. Jr., "A complex network approach for nanoparticle agglomeration analysis in nanoscale images," Journal of Nanoparticle Research, vol. 19, no. 2, pp. 65-73, 2017. 